International Journal of Pure and Applied Mathematics

Volume 107 No. $2 \quad 2016,457-477$

ISSN: 1311-8080 (printed version); ISSN: 1314-3395 (on-line version)

url: http://www.ijpam.eu

doi: 10.12732/ijpam.v107i2.15

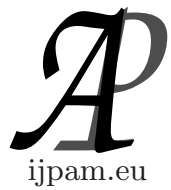

\title{
SIGMA-1-1 WELL-FOUNDED RELATIONS AND SET CHAINS
}

\author{
Martin Dowd \\ 1613 Wintergreen Pl. \\ Costa Mesa, CA 92626, USA
}

\begin{abstract}
Arbitrary well-founded relations are considered, generalizing constructions involving WPS's given in [9]. Some new facts concerning function chains and set chains are given, and a new axiom for set theory.
\end{abstract}

AMS Subject Classification: $03 \mathrm{e} 55$

Key Words: function chains, set chains, new axioms, generalized constructive ordinals

\section{Introduction}

In a series of papers $([2],[3],[4],[5],[6],[7])$ the author has "constructed" progressively longer chains of stationary sets of inaccessible cardinals. The existence of such chains is independent of ZFC (indeed the existence of an inaccessible cardinal is), so that the construction in fact yields a chain must be postulated. This gives a quantitative theory, justifying the addition of certain axioms to ZFC, stating that the cumulative hierarchy satisfies certain principles regarding its extendibility.

Throughout these constructions, the fact that the axiom holds in $V_{\kappa}$ if $\kappa$ is weakly compact has been observed; the stationary sets are in the enforceable filter. One goal of this research has been to obtain a weakly compact cardinal by making the stationary set chains long enough, using the principle of continuing to extend the cumulative hierarchy.

In [7] the set chains constructed involve the notion of a $\Sigma_{1}^{1}$ WPS. A WPS is a well-founded binary relation (WF). In this paper set chains are obtained from $\Sigma_{1}^{1}$ WF's, under a certain hypothesis, and various other facts of interest are

Received: November 30, 2015

Published: April 11, 2016 (c) 2016 Academic Publications, Ltd.

url: www.acadpubl.eu 
proved. Understanding the properties of $\Sigma_{1}^{1}$ WF's is of interest in the context of set chains, and in general.

For a cardinal $\kappa$ the three structures $L_{\kappa}, \mathrm{OS}_{\kappa}$, and $H_{\kappa}$, may be considered. In each the $\Sigma_{1}$ WF's, and various subclasses thereof, may be considered. If $V=L$ these all define the same ranks. It has been shown [12] that it is consistent that they do not.

Classes involving second order objects may be defined. These are the classes relevant to constructing stationary set chains. The classes of the previous paragraph for the cardinal $\kappa^{+}$provide characterizations of them. As will be seen, $\mathrm{OS}_{\kappa}$ has advantages over $H_{\kappa}$ in this role.

In [9] it is claimed that if $V=L$ the ranks of the $\mathcal{U} \Sigma_{1 l^{1}}^{1}$ WPS's (defined below) are the same as the ranks of the $\Sigma_{1}^{1}$ WPS's. The proof given is incorrect. As of this paper, this question is still open. Some results given here suggest that the claim may be false.

Let Card denote the class of cardinals, Inac the class of strongly inaccessible cardinals, and write $\operatorname{Inac}_{\kappa}$ for $\operatorname{Inac} \cap \kappa$. Let $\operatorname{Cf}(\alpha)$ denote the cofinality of an ordinal $\alpha$.

\section{Well-Founded Relations}

A well-founded relation (WF) on a set $S$ is a binary relation $\prec$ on $S$ such that for any function $f: \omega \mapsto S$ there is an $n$ such that $f(n+1) \nprec f(n)$ (i.e., there are no infinite descending chains). The rank or height $\Omega(x)$ of an element $x \in \operatorname{Fld}(\prec)$ is an ordinal, defined recursively as $\sup \{\Omega(y)+1: y \prec x\} . \Omega(\prec)$ is defined to be $\sup \{\Omega(x)+1: x \in \operatorname{Fld}(\prec)\}$, which is taken as 0 if $\prec$ is empty.

If $x \in \operatorname{Fld}(\prec)$ let $\prec_{x}$ denote $\prec \cap\left(x_{\prec} \times x_{\prec}\right)$ where $x_{\prec} \operatorname{denotes}\{y: y \prec x\}$. $\prec_{x}$ is well-founded and $\Omega\left(\prec_{x}\right)=\Omega(x)$.

Let $\Upsilon(\prec)$ denote the supremum of the lengths of the ascending chains in $\prec$. It is readily seen that $\Upsilon(\prec) \leq \Omega(\prec)$; it is well-known [16] that strict inequality can hold.

Recalling the definition from [7], a WPS on a set $S$ is a binary relation $\preceq$ on $S$ satisfying the following axioms:

T1. $A \preceq B \wedge B \preceq C \Rightarrow A \preceq C$

T2. $A \preceq B \Rightarrow A \preceq A$

T3. $A \preceq B \Rightarrow B \preceq B$

T4. $A \preceq A \wedge B \preceq B \Rightarrow(A \preceq B \vee B \preceq A)$

F. For all functions $f$ with domain $\omega$ there is an $n$ such that $f(n+1) \preceq$ $f(n) \Rightarrow f(n) \preceq f(n+1)$ 
The strict part is the relation $\prec$ where $A \prec B$ iff $A \preceq B \wedge \neg B \preceq A$; axiom $\mathrm{F}$ implies that it is well-founded. Let $\Omega(\preceq)=\Omega(\prec)$. For $x \in \operatorname{Fld}(\preceq)$ let $\preceq_{x}$ denote $\preceq \cap\left(x_{\prec} \times x_{\prec}\right)$; this is readily seen to be a WPS.

Theorem 1. For a WPS $\preceq, \Upsilon(\prec)=\Omega(\prec)$.

Proof. Let $\equiv$ be the relation " $x \leq y \wedge y \leq x$ ". It is readily seen that $\equiv$ is a congruence relation, the quotient $\prec / \equiv$ is a well-order, and $\Upsilon(\prec)=\Upsilon(\prec / \equiv$ )$=\Omega(\prec / \equiv)=\Omega(\prec)$.

A WOS (well-order on a subset) is a WPS where $A \preceq B \wedge B \preceq A \Rightarrow A=B$. In a WOS $\prec$ may be defined by " $A \preceq B \wedge A \neq B$ ". For a WOS $\preceq$ and an $x \in \operatorname{Fld}(\preceq), \preceq_{x}$ is a WOS. A WP (well-preorder) is a WPS where axioms T2T4 are replaced by $A \preceq B \vee B \preceq A$. A WO (well-order) is a WPS which is both a WOS and a WP. For a WO the rank is also called the order type.

\section{3. $\mathrm{OS}_{\kappa}$}

The structure $\mathrm{OS}_{\kappa}$ is defined in [7]; an earlier version, $K_{\kappa}^{s}$, may be found in [2]. To review the definition, let $L_{\mathrm{OS}}$ be the language with two sorts, Ord and Seq. Variables of Ord sort are denoted $\alpha, \beta$, etc., and those of Seq sort $s, t$, etc. The functions and relations are:

$0,1, \alpha+\beta, \alpha<\beta$,

$\operatorname{Dom}(s)$ (of sort Ord), $\operatorname{Elem}(s, \alpha)$ (of sort Ord),

and equality on Ord and on Seq. As in [2], $s(\alpha)$ may be written for $\operatorname{Elem}(s, \alpha)$ and $|s|$ for $\operatorname{Dom}(s)$.

The version in [7] has also the function symbol $\operatorname{Rstr}(s, \alpha, \beta)$ (of sort Seq). The graph of this function has a $\Delta_{0}$ definition in the smaller language, namely, $P \wedge \forall \gamma<|t|(t(\gamma)=s(\alpha+\gamma) \vee \neg P \wedge t=\emptyset$ where $P$ is $\beta=\alpha+|t| \wedge \beta<|s|$.

Bounded quantifiers in $L_{\mathrm{OS}}$ are those of the form $\forall \gamma<\beta$ or $\exists \gamma<\beta$ where $\beta$ is a term. $\Delta_{0}, \Sigma_{1}$, and $\Pi_{1}$ formulas are defined as usual, where free variables and unbounded quantifiers may be of either sort.

For $\kappa \in$ Card $\mathrm{OS}_{\kappa}$ is the structure for $L_{\mathrm{OS}}$ where Ord is interpreted as $\kappa$ and Seq as $\{f: \alpha \mapsto \kappa: \alpha<\kappa\}$. The functions and relations have their self-evident interpretations; Elem has value 0 if the index is out of range.

Various facts of interest can be proved to hold in $\mathrm{OS}_{\kappa}$ for any $\kappa$. Indeed, an axiom system $A_{\mathrm{OS}}$ is given in [7], and basic facts can be proved in it. For the version above, the axiom for Rstr may be omitted.

Lemma 2. a. The $\Sigma_{1}$ predicates are closed under bounded quantification. 
b. If $G:$ Seq $\mapsto$ Ord is a $\Sigma_{1}$ function then there is a $\Sigma_{1}$ function $F:$ Ord $\mapsto$ Ord such that for all $\alpha F(\alpha)=G(F \uparrow \alpha)$.

Proof. This is lemma 21 of [7]. An examination of the proof shows that it can be carried out in $A_{\mathrm{OS}}$.

Note also that $\alpha<\omega$ is a $\Delta_{0}$ predicate, and it is not difficult to show that Peano Arithmetic is interpretable in $A_{\mathrm{OS}}$. The sort "integer" may be added to the language, without changing the complexity of formulas; $n, m, \ldots$ will be used to denote integers.

The rank of a WF on Seq is less than $\left(\kappa^{<\kappa}\right)^{+}$. That of a WF on Ord is less than $\kappa^{+}$. When $\kappa=\lambda^{+}$the bounds become $\left(\left(\lambda^{+}\right)^{\lambda}\right)^{+}=\left(2^{\lambda}\right)^{+}$and $\lambda^{++}$

The language $L_{\mathrm{OS}}^{f}$ is also of interest; some consideration may be found in [9]. This adds second order function variables; these take an ordinal argument and produce an ordinal value. $\mathrm{OS}_{\kappa}$ may be considered as a structure for this language by interpreting second order function symbols as elements of $\mathcal{N}$ where $\mathcal{N}$ denotes $\kappa^{\kappa}$. As above, $t=\operatorname{Rstr}(F, \alpha, \beta)$ is a $\Delta_{0}^{0}$ definable predicate.

Say that a formula of $L_{\mathrm{OS}}^{f}$ is $\Sigma_{1}^{1 P}$ if it is of the form $\exists \vec{F} \phi$ where $\phi$ is $\Pi_{1}^{0}$.

Theorem 3. In the $\Sigma_{1}^{1 P}$ formulas over $O S_{\kappa}$ for $\kappa \in$ Card, multiple second order existential quantifiers may be combined. The $\Sigma_{1}^{1 P}$ predicates are closed under quantification over variables of Ord sort, and existential quantification over variables of Seq sort.

Proof. A formula $\exists F_{0} \exists F_{1} \phi\left(F_{0}, F_{1}\right)$ where $\phi$ is $\Pi_{1}^{0}$ may be written as $\exists G \phi^{\prime}$ where $\phi^{\prime}$ is obtained from $\phi$ by replacing $F_{i}(\tau)$ by $G(\tau+\tau+i)$ (where $\tau$ is a term). A formula $\exists \alpha \exists F \phi(\alpha, F)$ may be written as $\exists G \phi^{\prime}$ where $\phi^{\prime}$ is obtained from $\phi$ by replacing $\alpha$ by $G(0)$ and $F(\tau)$ by $G(1+\tau)$. A formula $\forall \alpha \exists F \phi(\alpha, F)$ may be written as $\exists G \forall \alpha \phi^{\prime}$ where $\phi^{\prime}$ is obtained from $\phi$ by replacing $F(\tau)$ by $G\left(J_{0}(\alpha, \tau)\right)$ where $J_{0}$ is the Godel pairing function. $\phi^{\prime}$ is $\Pi_{1}^{1}$ by lemma 21.a of [7]. A formula $\exists s \exists F \phi(s, F)$ may be written as $\exists G \phi^{\prime}$ where $\phi^{\prime}$ is obtained from $\phi$ by replacing $s$ by $\operatorname{Rstr}(G, G(0), 1+G(0))$ and $F(\tau)$ by $G(\tau+1+G(0))$.

Theorem 4. Suppose $\kappa$ is regular uncountable and $\kappa^{<\kappa}=\kappa$. The $\Sigma_{1}^{1 P}$ predicates are closed under universal quantification over variables of Seq sort.

Proof. Let $\beta_{1}=|s|, \beta_{2}=\sup _{\gamma<\beta_{1}} s(\gamma), \beta_{3}=\sup \left(\beta_{1}, \beta_{2}\right)$, and $\beta(s)=$ $\sum_{\gamma \leq \beta_{3}} \gamma \cdot \gamma^{\gamma}$. Let $C\left(H_{1}, H_{2}\right)$ denote $\forall s \exists \alpha<\beta(s) s=\operatorname{Rstr}\left(H_{2}, H_{1}(\alpha), H_{1}(\alpha+\right.$ $1))$. Let $\alpha=X\left(H_{1}, H_{2}, s\right)$ denote $s=\operatorname{Rstr}\left(H_{2}, H_{1}(\alpha), H_{1}(\alpha+1)\right) \wedge \forall \beta<$ $\alpha s \neq \operatorname{Rstr}\left(H_{2}, H_{1}(\beta), H_{1}(\beta+1)\right)$. A formula $\forall s \exists F \phi(\alpha, F)$ may be written as 
$\exists H_{1} \exists H_{2} \exists G\left(C\left(H_{1}, H_{2}\right) \wedge \forall s \phi^{\prime}\right.$ where $\phi^{\prime}$ is obtained from $\phi$ by replacing $F(\tau)$ by $G\left(J_{0}\left(X\left(H_{1}, H_{2}, s\right), \tau\right)\right)$.

The analog of this theorem holds for $\Sigma_{1}^{1 L}$ predicates (defined below); proofs may be found in [13],[9].

Lemma 5. a. For any $\kappa \in$ Card, a predicate $R \subseteq \mathcal{N}^{k}$ defined in $O S_{\kappa}$ by a $\Sigma_{1}^{0}$ formula of $L_{O S}^{f}$ is open.

b. If $\kappa$ is regular uncountable and $\kappa^{<\kappa}=\kappa$ the converse holds.

Proof. Part a follows by theorem 11 of [9]. For part b, suppose $R \subseteq \mathcal{N}^{k}$ is open. $R$ can be specified by a subset $D_{R}$ of $\left(\kappa^{<\kappa}\right)^{k}$. Since $\kappa^{<\kappa}=\kappa, D_{R}$ can be coded as an element $F_{R}$ of $\mathcal{N}$ using a "separator" value. Then $G \in R$ iff "there is an $s$ such that $F_{R}$ witnesses $s \in D_{R}$ and $s$ is a prefix of $G$ "; this statement is is $\Sigma_{1}^{0}$.

Note that part $\mathrm{b}$ answers a question noted following theorem 15 of [9], and renders that theorem irrelevant.

\section{Classes over $\kappa$}

In this section, let $\kappa$ be a cardinal. Let $L_{\in}$ be the language of set theory. Let $L_{\kappa}$ be the $\kappa$ th level of the constructibility hierarchy, considered as a structure for $L_{\epsilon}$. Let $H_{\kappa}$ be sets whose transitive closure has cardinality less than $\kappa$, considered as a structure for $L_{\in}$.

Classes of relations $C$ will be defined, on a domain $D$ (i.e., subsets of $D^{k}$ for some $k$ ). If defined by formulas the free variables are restricted to $D$; parameters are also in $D$, unless otherwise specified.

The following classes are defined.

$C_{\nvdash}: D=\kappa, \Sigma_{1}$ in $L_{\in}$ over $L_{\kappa}$.

$C_{\nVdash}: D=\operatorname{Ord}, \Sigma_{1}$ in $L_{\mathrm{OS}}$ over $\mathrm{OS}_{\kappa}$.

$C_{\nVdash \approx}: D=\kappa, \Sigma_{1}$ in $L_{\in}$ over $H_{\kappa}$.

$C_{\not \nvdash}: D=\mathrm{Seq}, \Sigma_{1}$ in $L_{\mathrm{OS}}$ over $\mathrm{OS}_{\kappa}$.

$C_{\not \neq \approx}: D=\kappa^{<\kappa}, \Sigma_{1}$ in $L_{\in}$ over $H_{\kappa}$.

$C_{\nvdash \mathbb{H}}: D=\kappa^{<\kappa}, \Sigma_{1}$ in $L_{\in}$ over $H_{\kappa}$ with parameters in $H_{\kappa}$.

$C_{\nVdash}: D=H_{\kappa}, \Sigma_{1}$ in $L_{\in}$ over $H_{\kappa}$.

Say that class $C$ is transformable to class $C^{\prime}$, written $C \rightsquigarrow C^{\prime}$, if there is an injection $j: D \mapsto D^{\prime}$ which induces a map from $C$ to $C^{\prime}$. If $C$ is a class of WF's let $\Upsilon(C)=\sup (\Upsilon(\prec)): \prec \in C\}$. For a class of relations $C$, let $\Upsilon(C)$ denote $\Upsilon\left(C_{\prec}\right)$ where $C_{\prec}$ is the WF's of $C$. Note that if $C \rightsquigarrow C^{\prime}$ then $\Upsilon(C) \leq \Upsilon\left(C^{\prime}\right)$. 
Let $C_{\nVdash}^{\prime}$ be as $C_{\nVdash}$, but with unrestricted free variables and parameters. Because there is a $\Sigma_{1}$ bijection between $\kappa$ and $L_{\kappa}$, these may be used interchangeably.

Let $F_{f}, \in_{f}$, and $=_{f}$ be as in [9]. $L_{\in}$ may be interpreted in $L_{\text {OS }}$, by interpreting $\in$ as $\in_{f}$ and $=$ as $=_{f}$. Denote this interpretation as $I_{f}$.

Lemma 6. For any formula $\phi$ of $L_{\in}, \phi\left(F_{f}\left(\alpha_{1}\right), \ldots, F_{f}\left(\alpha_{k}\right)\right)$ holds in $L_{\kappa}$ iff $\phi^{I_{f}}(\vec{\alpha})$ holds in $O S_{\kappa}$. If $\phi$ is $\Delta_{0}$ then $\phi^{I_{f}}$ is $\Delta_{1}$.

Proof. The first claim follows by induction on $\phi$. The second follows by induction, using the fact, noted in [9], that $\epsilon_{f}$ and $=_{f}$ are $\Delta_{1}$; and lemma 12 of $[9]$.

There is also an interpretation of $L_{\mathrm{OS}}$ in $L_{\in}$. Ord is interpreted as the ordinals, and Seq as functions with domain an ordinal and ordinal values. Denote this interpretation as $I_{\in}$. The formulas for the symbols of $L_{\mathrm{OS}}$ are $\Delta_{0}$, except the graph of $\alpha+\beta$, which is $\Delta_{1}$, and so $\Delta_{0}$ formulas translate to $\Delta_{1}$ formulas.

\section{Theorem 7.}

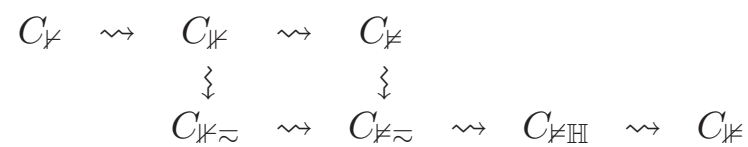

Thus, $\Upsilon\left(C_{\nvdash}\right) \leq \Upsilon\left(C_{\nVdash}\right) \leq \Upsilon\left(C_{\not \models}\right) \leq \Upsilon\left(C_{\nVdash}\right)$.

Proof. Let $\phi$ be a $\Sigma_{1}$ formula defining a WF in $L_{\kappa}$. By lemma $6, \phi^{I_{f}}$ defines a $\Sigma_{1}$ WF in $\mathrm{OS}_{\kappa}$, of the same rank. Further $\phi^{I_{f}}$ has ordinal arguments, and ordinal parameters can be chosen. This proves $C_{\nvdash} \rightsquigarrow C_{\nVdash}$. $C_{\nVdash} \rightsquigarrow C_{\not \models}$ follows by interpreting ordinals as length 1 sequences. $C_{\nVdash} \rightsquigarrow C_{\nVdash \approx}$ and $C_{\not \models} \rightsquigarrow C_{\not \models \approx}$ follow using $I_{\in} . C_{\nVdash z} \rightsquigarrow C_{\not \models \approx} \rightsquigarrow C_{\not \models \mathbb{H}} \rightsquigarrow C_{\nVdash}$ follows trivially.

As will be seen below, more can be shown if $\kappa$ is a successor cardinal.

Theorem 8. If $V=L$ then $\Upsilon\left(C_{\nvdash}\right)=\Upsilon\left(C_{\nVdash}\right)$.

Proof. This follows because if $V=L$ then $H_{\kappa}=L_{\kappa}$ (corollary 5.2.7 of $[10])$.

By cardinality, $\Upsilon\left(C_{\nVdash}\right)<\kappa^{+}$. As will be seen below, for suitable $\kappa, \Upsilon\left(C_{\not \models}\right) \geq$ $\kappa^{+}$is consistent. 


\section{Coding Formulas}

Formulas with parameters from Seq can be coded as elements of Seq. Formulas with parameters from Ord can be coded as elements of Ord. Both codings will be given. Each involves a coding of finite sequences.

In Seq a sequence (or list) of elements of ordinal length can readily be coded as an element; this has uses, and finite sequences are a special case. The list $s_{\gamma}: \gamma<\delta$ may be coded as $\delta \frown \eta_{0} \ldots \frown s_{0} \ldots$, where $\eta_{\gamma}=\sum_{\zeta \leq \gamma}\left|s_{\zeta}\right|$. The statement that $s$ occurs in a list $l$ is $\Delta_{0}$. This coding may be used for a pairing function on sequences. Let $J_{S}(s, t)$ denote the code for the pair $s, t$; this function has a $\Delta_{0}$ graph.

In Ord a finite sequence of elements can be coded as an element using the Godel pairing function. The sequence $\alpha_{i}: i<n$ may be coded as $J_{0}\left(n, J_{0}\left(\alpha_{1}, \ldots\right.\right.$, $\left.\left.J_{0}\left(\alpha_{n-1}, n\right) \ldots\right)\right)$. Functions manipulating these codes are $\Delta_{1}$.

It is well-known that a formula $\phi$ without parameters can be coded as an integer $\ulcorner\phi\urcorner$, so that syntactic functions are $\Delta_{1}$ in the language of arithmetic, and hence in $L_{\mathrm{OS}}$ over $\mathrm{OS}_{\kappa}$ for $\kappa \in$ Card.

Suppose $\phi$ is a formula and $x_{1}, \ldots, x_{k}$ are its free variables in alphabetic order. Suppose $v_{i}$ is a value of the sort of $x_{i}$. Let $l$ be the code for $v_{1}, \ldots, v_{k}$. The sentence with parameters $\phi\left(v_{1}, \ldots, v_{k}\right)$ can be coded by replacing $l(0)=k$ by $\ulcorner\phi\urcorner$. This may be done for either the codes over Seq or the codes over Ord. Over Seq, a value $v_{i}$ of sort Ord may be considered a sequence of length 1 , and if $k=0$ the code is a sequence of length 1 . Over Ord, if $k=0$ the code may be taken as $J_{0}(\ulcorner\phi\urcorner, 0)$.

The code just described will be denoted $\left\ulcorner\phi\left(v_{1}, \ldots, v_{k}\right)\right\urcorner$. Let $\Phi$ denote the sentences with parameters and $\mathcal{I}$ their codes (although as has been observed by some authors the latter can be used for the former).

Theorem 9. Over Seq or Ord, there is a $\Delta_{1}$ formula $\operatorname{Tru}_{0}(c)$ such that for any $\kappa \in C$ ard, in $O S_{\kappa}$, for any $\Delta_{0} \phi(\vec{v}) \in \Phi, \phi(\vec{v}) \Leftrightarrow \operatorname{Tr}_{0}(\ulcorner\phi(\vec{v})\urcorner)$.

Proof. The proof will use "partial truth assignments"; see definition 1.71 of [11] for a related concept. A partial truth assignment is a list in 4 parts, a list of terms, a list of their values, a list of formulas, and a list of their truth values, satisfying certain restrictions. These restrictions may be stated by a $\Delta_{1}$ formula $\operatorname{PTA}(a)$.

PTA may be broken into cases; one example will be given, and the rest left to the reader. For all terms $t=t_{1}+t_{2}$ occurring in $a$, for $i=1,2$, either $t_{i}$ is a variable or $t_{i}$ (with value list adjusted) occurs in $a$; in addition $v=v_{1}+v_{2}$ where $v$ is the value of $t$ and for $i=1,2 v_{i}$ is the value of $t_{i}$. 
$\operatorname{Tru}_{0}(c)$ may be stated in $\Sigma_{1}$ form as "for some $a, \operatorname{PTA}(a)$ and $c$ occurs in $a$ and $c$ has value 1 in $a$ ". $\operatorname{Tru}_{0}(c)$ may be stated in $\Pi_{1}$ form as "for all $a$, if $\operatorname{PTA}(a)$ and $c$ occurs in $a$ then $c$ has value 1 in $a "$.

Corollary 10. Over Seq or Ord, there is a $\Sigma_{1}$ formula Tru(c) such that for any $\kappa \in$ Card, in $O S_{\kappa}$, for any $\Sigma_{1}$ sentence with parameters $\phi(\vec{v}), \phi(\vec{v}) \Leftrightarrow$ $\operatorname{Tru}(\ulcorner\phi(\vec{v})\urcorner)$.

Proof. This follows by a standard argument.

In $L_{\kappa}$ (or $H_{\kappa}$, or any admissible set) an ordered pair of elements may be coded as the ordered pair in the set. Sentences with parameters may be coded as in the case of $\mathrm{OS}_{\kappa}$ with parameters from Ord. It is a standard fact of admissible set theory that there is a $\Sigma_{1}$ predicate $\operatorname{Tru}(c)$ which holds for a code $c$ of a $\Sigma_{1}$ sentence with parameters iff the sentence is true (see proposition V.1.8 of [1] for example). Although not the usual method, the method of corollary 10 can be used to prove this fact. It is only necessary to observe that in an admissible set $A$, any $\Delta_{0}$ sentence with parameters has a partial truth assignment in $A$.

A formula $\phi\left(\vec{\alpha}_{p}, \vec{\alpha}, \vec{s}_{p}, \vec{s}\right)$, where the free variables are listed in alphabetic order, with values $v_{O i}$ for the variables $\alpha_{p i}$, and values $v_{S i}$ for the variables $s_{p i}$, may be coded by replacing $l(0)$ by $\ulcorner\phi\urcorner$ in the code $l$ for the list $\vec{v}_{O}, \vec{v}_{S}$. Similar remarks hold over Ord.

In an expression $\ulcorner\phi\urcorner$ a parameter of $\phi$ depending on a value $\alpha$ may be denoted $\stackrel{\circ}{\alpha}$.

\section{Constructive Ordinals in Seq}

This section provides adaptations of various facts about constructive ordinals (as found in [15] for example) to $\mathrm{OS}_{\kappa}$ for $\kappa \in$ Card. Essentially the same development can be carried out for $H_{\kappa}$, but this is omitted.

Let $\Phi_{1}$ denote the $\Sigma_{1}$ formulas with no parameters and a single free variable of sort Seq. Let $\mathcal{I}_{1} \subseteq \omega$ denote the integers which code elements of $\Phi_{1}$. For $e \in \mathcal{I}_{1}$ let $\phi_{e}$ denote the formula coded by $e$ and let $W_{e}$ denote the subset of Seq defined by $\phi_{e}$.

Let $\mathcal{F}_{0}$ denote the functions $f$ : Seq $\mapsto$ Seq which are total and whose graph is $\Sigma_{1}\left(\right.$ and hence $\left.\Delta_{1}\right)$ without parameters in $L_{\mathrm{OS}}$. Let $\mathcal{F}_{1}=\left\{f \in \mathcal{F}_{0}: f\left[\mathcal{I}_{1}\right] \subseteq\right.$ $\left.\mathcal{I}_{1}\right\}$.

Theorem 11. a. For any $e_{0} \in \mathcal{I}_{1}$ there is an $e_{1} \in \mathcal{I}_{1}$ such that $W_{e_{1}}(s) \Leftrightarrow$ $W_{e_{0}}\left(J_{S}\left(e_{1}, s\right)\right)$ for all $s$. 
b. Suppose $f \in \mathcal{F}_{1}$; then there is an $e \in \mathcal{I}_{1}$ such that $W_{f(e)}=W_{e}$.

c. Suppose $<\subseteq$ Seq $\times$ Seq is well-founded and $f \in \mathcal{F}_{1}$. Suppose $\forall e \in \mathcal{I}_{1} \forall x \in$ $\operatorname{Fld}(<)\left(\forall x^{\prime}<x \exists ! y W_{e}\left(J_{S}\left(x^{\prime}, y\right)\right) \Rightarrow \exists ! y W_{f(e)}\left(J_{S}(x, y)\right)\right)$. Suppose $e \in \mathcal{I}_{1}$ is such that $W_{f(e)}=W_{e}$. Then $\forall x \in \operatorname{Fld}(<) \exists ! y W_{e}\left(J_{S}(x, y)\right)$.

Proof. For part a, let $\operatorname{Tru}_{1}(e, s)=\operatorname{Tru}\left(F\left(e_{1}, s\right)\right)$ where for $e \in \mathcal{I}_{1} F(e, s)$ equals $\left\ulcorner\phi_{e}(s)\right\urcorner$, that is, $e \frown|s| \neg s$. Let $f \in \mathcal{F}_{1}$ be such that for any $e \in \mathcal{I}_{1} f(e)=$ $\left\ulcorner\operatorname{Tru}_{1}\left(N_{e}, J_{s}\left(N_{e}, s\right)\right)\right\urcorner$ where $N_{e}$ is the numeral for $e$ and $s$ is the free variable of $\phi_{e}$; note that $W_{f(e)}(s) \Leftrightarrow W_{e}\left(J_{s}(e, s)\right)$. Let $e_{2}=\left\ulcorner\phi_{e_{0}}\left(J_{S}\left(f\left(P_{1}(t)\right), P_{2}(t)\right)\right)\right\urcorner$ where $P_{1}, P_{2}$ are the "projection functions" for $J_{S}$; note that $W_{e_{2}}\left(J_{S}(e, s)\right) \Leftrightarrow$ $W_{e_{0}}\left(J_{S}\left(f\left(e_{0}\right), s\right)\right)$. Let $e_{1}=f\left(e_{2}\right)$. Direct computation verifies that the requirement on $e_{1}$ is satisfied.

For part b, let $e_{0}=\left\ulcorner\operatorname{Tru}_{1}\left(f\left(P_{1}(t)\right), P_{2}(t)\right)\right\urcorner$; note that $W_{e_{0}}\left(J_{S}\left(e_{1}, s\right)\right) \Leftrightarrow$ $W_{f\left(e_{1}\right)}(s)$. Now choose $e_{1}$ as in part a.

For part c, if there is an $x \in \operatorname{Fld}(<)$ such that $\neg \exists$ ! $\left.y W_{e}\left(J_{S}(x, y)\right)\right)$ let $x$ be a minimal such. Then $\exists ! y W_{f(e)}\left(J_{S}(x, y)\right)$, whence $\exists ! y W_{e}\left(J_{S}(x, y)\right)$, a contradiction.

Let $<_{O}$ be the predicate on Seq $\times$ Seq, which is the least predicate satisfying the following conditions, where $O$ denotes $\operatorname{Fld}\left(<_{O}\right)$.

1. $\emptyset<_{O} s$ where $\operatorname{Dom}(s)=1$ and $s(0)=0$.

2. If $s \in O$ then $s<_{O} 0 \frown s$.

3. Suppose $\theta(\gamma, t, \vec{p})$ is a $\Sigma_{1}$ formula with parameters $\vec{p}$ defining a total function $f$ : Ord $\mapsto O$ such that $\alpha<\beta \Rightarrow f(\alpha)<_{O} f(\beta)$, and let $s=1 \frown\ulcorner\theta(\gamma, t, \vec{p})\urcorner$. Then for all $\alpha, f(\alpha)<_{O} s$.

4. Suppose $\delta$ is a limit ordinal and $\theta(\gamma, t, \vec{p})$ is a $\Sigma_{1}$ formula with parameters $\vec{p}$ defining a predicate which is a total function $f: \delta \mapsto O$ when restricted to arguments $\alpha<\delta$, such that $\alpha<\beta<\delta \Rightarrow f(\alpha)<_{O} f(\beta)$, and let $s=2 \frown \delta \frown\ulcorner\theta(\gamma, t, \vec{p})\urcorner$. Then for all $\alpha<\delta, f(\alpha)<_{O} s$.

$5 .<_{O}$ is transitive.

In cases 3 and 4 call $f$ the defining function.

Theorem 12. a. $<_{O}$ is well-founded.

b. If $t_{1}<_{O} s$ and $t_{2}<_{O} s$ then $t_{1}=t_{2}$ or $t_{1}<_{O} t_{2}$ or $t_{2}<_{O} t_{1}$.

c. If $0 \frown s \in O$ then there is no $t \in O$ such that $s<_{O} t<_{O} 0 \frown s$.

d. If $1 \frown s \in O$ where $f$ is the defining function then there is no $t \in O$ such that for all $\alpha f(\alpha)<_{O} t<_{O} 1 \frown s$.

e. If $2 \frown \delta \frown s \in O$ where $f$ is the defining function then there is no $t \in O$ such that for all $\alpha<\delta f(\alpha)<_{O} t<_{O} 2 \frown \delta \frown s$. 
Proof. Let $O_{0}=\{\emptyset\}$. Let $O_{\alpha+1}=O_{\alpha}$ together with the elements added by clauses 2-4 of the definition. For $\alpha \in \operatorname{Lim}$ let $O_{\alpha}=\cup_{\beta<\alpha} O_{\beta}$. Define the rank of $s \in O$ to be the least $\alpha$ such that $s \in O_{\alpha}$; then $\alpha=\beta+1$ for some $\beta$. It is readily seen that if $u<_{O} s$ then there is a $t \in O_{\beta}$ such that $u \leq_{O} t<_{O} s$ where $t<_{O} s$ follows by clauses $2-4$. The theorem follows.

By the theorem, for $s \in O$ the ordinal $\Omega(s)$ may be defined to be $\Omega\left\{\left\langle t_{1}, t_{2}\right\rangle\right.$ : $\left.t_{1}<_{O} t_{2}\right\}$. Further, letting $f$ be the defining function, $\Omega(\emptyset)=0, \Omega(0 \frown s)=$ $\Omega(s)+1, \Omega(1 \frown s)=\sup _{\alpha} \Omega(f(\alpha))$, and $\Omega(2 \frown \delta \frown s)=\sup _{\alpha<\delta} \Omega(f(\alpha))$.

Theorem 13. There is a function $p \in \mathcal{F}_{0}$ such that for $s \in O, p(s) \in \mathcal{I}_{1}$ and $W_{p(s)}=\{t: t<o s\}$.

Proof. Let $f \in \mathcal{F}_{0}$ be such that for $e \in \mathcal{I}_{1}, W_{f(e)}\left(J_{S}(s, t)\right)$ iff $t=\ulcorner\theta\urcorner$ where $\theta \in \Phi_{1}$ is a formula defined by cases as follows; $r$ is used for the free variable of $\theta$.

If $s=\emptyset$ then $\theta$ is $r \neq r$.

If $s=0 \frown s^{\prime}$ then $\theta$ is $r=s^{\prime} \vee \exists t^{\prime}\left(\phi_{e}\left(J_{S}\left(s^{\prime}, t^{\prime}\right)\right) \wedge \operatorname{Tru}_{1}\left(t^{\prime}, r\right)\right)$.

If $s=1 \frown s^{\prime}$ then $\theta$ is $\exists \alpha \exists s_{2} \exists t_{2}\left(\operatorname{Tru}_{2}\left(s^{\prime}, \alpha, s_{2}\right) \wedge \phi_{e}\left(J_{S}\left(s_{2}, t_{2}\right)\right) \wedge \operatorname{Tru}_{1}\left(t_{2}, r\right)\right)$ where $\mathrm{Tru}_{2}$ is a suitable variation of Tru.

If $s=2 \frown \delta \frown s^{\prime}$ then $\theta$ is as in the previous case, except $\exists \alpha$ is replaced by $\exists \alpha<\delta$.

Note that $\ulcorner\theta\urcorner$ is actually a formula defining this element of Seq from $s$, etc. Then $f$ satisfies the hypotheses of theorem 11.c. Let $p$ be the function whose graph is defined by $W_{e}$ for $e$ as in the conclusion of theorem 11.c. The theorem follows.

Theorem 14. There is a function $q \in \mathcal{F}_{0}$ such that for $s \in O, q(s) \in \mathcal{I}_{1}$ and $W_{q(s)}=\left\{J_{S}\left(t_{1}, t_{2}\right): t_{1}<_{O} t_{2}<_{O} s\right\}$.

Proof. The proof is the same as that of the preceding theorem, except that in the case $s=0 \frown s^{\prime} \theta$ is $r=s^{\prime} \vee \exists t^{\prime}\left(\phi_{e}\left(J_{S}\left(s^{\prime}, t^{\prime}\right)\right) \wedge \operatorname{Tru}_{1}\left(t^{\prime}, r\right)\right)$.

Theorem 15. There is a function $s_{1}+o s_{2}$ with $\Sigma_{1}$ graph, such that if $s_{1}, s_{2} \in O$ then $s_{1}+O s_{2} \in O$ and $\Omega\left(s_{1}+O s_{2}\right)=\Omega\left(s_{1}\right)+\Omega\left(s_{2}\right)$; and also if $s_{1}+O s_{2} \in O$ then $s_{1}, s_{2} \in O$.

Proof. For convenience write $W_{e}(\vec{x})$ for $W_{e}(F(\vec{x}))$ where $F$ is an appropriate sequence coding function. Let $\perp$ denote the sequence $s$ of length 1 where $s(0)=3$. Let $f \in \mathcal{F}_{0}$ be such that for $e \in \mathcal{I}_{1}, \Phi_{f(e)}\left(s_{1}, s, t\right)$ satisfies the following clauses.

$s=\emptyset: t=s_{1}$. 
$s=0 \frown s^{\prime}: \exists t^{\prime}\left(\phi_{e}\left(s_{1}, s^{\prime}, t^{\prime}\right) \wedge\left(t^{\prime}=\perp \wedge t=\perp \vee t=0 \frown t^{\prime}\right)\right)$.

$s=1 \frown s^{\prime}$ : if $s^{\prime}=\ulcorner\psi(\vec{q}, \alpha, y)\urcorner$ then $t=1 \frown t^{\prime}$ where $t^{\prime}=\left\ulcorner\exists y^{\prime}\left(\psi\left(\vec{q}, \alpha, y^{\prime}\right) \wedge\right.\right.$ $\left.\left.\phi_{e}\left(s_{1}, y^{\prime}, t\right)\right)\right\urcorner$. else $t=\perp$.

$s=2 \frown \delta \frown s^{\prime}$ : as in the previous case, except $t=2 \frown \delta \frown t^{\prime}$.

$s(0) \notin\{0,1,2\}: t=\perp$.

Let $e$ be as in the conclusion of theorem 11.c; then $+_{O}$ has graph $W_{e}$.

Other facts as in theorem I.3.4 of [15] also follow. Note that for $s \in O$ $\Omega(s)<\kappa^{++}$, so in view of the remarks following theorem 8 , in some models, this version of $O$ does not represent every $\Sigma_{1}$ WF on Seq.

\section{Constructive Ordinals in Ord}

The development of the previous section can be carried out using formulas with free variables and parameters in Ord. For convenience the same notation is used. Following is a list of changes which are needed. The following changes are needed for theorem 11 .

- Free variables $s, t, \ldots$ are changed to $\alpha, \beta, \ldots$.

- $J_{0}$ is used rather than $J_{S}$.

- Formulas of $\Phi_{1}$ have a single free variable of sort Ord.

- $\mathcal{I}_{1}$ is unchanged.

- $\mathcal{F}_{0}$ is the $f$ : Ord $\mapsto$ Ord which are total and whose graph is $\Sigma_{1}$.

- $\mathcal{F}_{1}$ is as before.

- In theorem 11.c $<\subseteq$ Ord $\times$ Ord.

- In the proof of theorem 11.a, $F(e, s)$ equals $\left\ulcorner\phi_{e}(s)\right\urcorner$, that is, $J_{0}(e, s)$.

Let $<_{O}$ be the predicate on $\operatorname{Ord} \times$ Ord, which is the least predicate satisfying the following conditions, where $O$ denotes $\operatorname{Fld}\left(<_{O}\right)$.

1. $0<_{O} 1$.

2. If $\alpha \in O$ then $\alpha<_{O} \alpha \cdot 4+1$.

3. Suppose $\theta(\gamma, \beta, \vec{\pi})$ is a $\Sigma_{1}$ formula with parameters $\vec{\pi}$ defining a total function $f$ : Ord $\mapsto O$ such that $\alpha<\beta \Rightarrow f(\alpha)<_{O} f(\beta)$, and let $\alpha=\ulcorner\theta(\gamma, \beta, \vec{\pi})\urcorner \cdot 4+2$. Then for all $\gamma, f(\gamma)<_{O} \alpha$.

4. Suppose $\delta$ is a limit ordinal and $\theta(\gamma, \beta, \vec{\pi})$ is a $\Sigma_{1}$ formula with parameters $\vec{\pi}$ defining a predicate which is a total function $f: \delta \mapsto O$ when restricted to arguments $\gamma<\delta$, such that $\alpha<\beta<\delta \Rightarrow f(\alpha)<_{O} f(\beta)$, and let $\alpha=J_{0}(\ulcorner\theta(\gamma, \beta, \vec{\pi})\urcorner, \delta) \cdot 4+3$. Then for all $\gamma<\delta, f(\gamma)<_{O} \alpha$.

5. $<_{O}$ is transitive.

Theorems 13, 14, 15, and the properties of $\Omega$ hold, with the following changes. Free variables $s, t, \ldots$ are changed to $\alpha, \beta, \ldots$ Cases $0^{\frown} s, 1^{\frown} s$, 
and $2 \frown \delta \frown s$ are changed to $\alpha \cdot 4+1, \alpha \cdot 4+2$, and $J_{0}(\alpha, \delta) \cdot 4+3$. In the proof of theorem $15, \perp$ denotes 4 .

\section{Constructive Ordinals in $L_{\kappa}$}

Because $L_{\kappa}$ is a "recursively listed" admissible set (see [1]), constructive ordinals in $L_{\kappa}$ for $\kappa \in$ Card may be taken as elements of either $\kappa$ or $L_{\kappa}$. Choosing them in $\kappa$ makes the development more similar to that of the preceding section. In particular, the same sentence coding may be used. The following changes are needed for theorem 11.

- Free variables are ordinals $\alpha, \beta, \ldots$ (i.e., restricted to range over Ord), and parameters are in $\kappa$.

- Formulas of $\Phi_{1}$ have a single Ord free variable.

- $\mathcal{I}_{1}, \mathcal{F}_{0}$, and $\mathcal{F}_{1}$ are as before.

- In theorem 11.c $<\subseteq \kappa \times \kappa$.

$<_{O}$ on Ord $\times$ Ord is defined as in the previous section. Theorems 13, 14, 15 , and the properties of $\Omega$ hold as before.

Let $\mathcal{I}_{1 p}$ be the codes of the $\Sigma_{1}$ formulas with ordinal parameters and an ordinal free variable. Let $\phi_{\eta}$ be the formula with code $\eta$ and $W_{\eta}$ the set defined by $\phi_{\eta}$.

Theorem 16. There is a function $g \in \mathcal{F}_{0}$ such that for $\eta \in \mathcal{I}_{1 p}$, if $W_{\eta} \subseteq O$ then $g(\eta) \in O$, and for all $\alpha \in W_{\eta}, \Omega(\alpha)<\Omega(g(\eta))$.

Proof. In the integer case, this is lemma I.4.1 of [15]. Suppose $\phi_{\eta}$ is $\exists x \psi(x, \alpha, \pi)$ (let $g(\eta)=4$ if $\phi$ is not of this form). Let $r$ be the function where $r(\gamma)$ equals $P_{2}(\gamma)$ if $\psi\left(F_{f}\left(P_{1}(\gamma)\right), P_{2}(\gamma), \pi\right)$, else 0 . Let $\theta(\alpha, \beta)$ be the formula $\exists s \theta^{\prime}(s, \alpha, \beta)$ where $\theta^{\prime}$ states the following.

$|s|=\alpha+1, s(\alpha)=\beta$, and for $\gamma \leq \alpha$ the following hold.

$s(0)=r(0)$.

If $\gamma=\gamma^{\prime}+1$ then $s(\gamma)=s\left(\gamma^{\prime}\right)+o r(\gamma)$.

If $\gamma \in \operatorname{Lim}$ then $s(\gamma)=\left(J_{0}(\ulcorner\stackrel{\circ}{s}(\zeta)=\xi\urcorner, \gamma) \cdot 4+3\right)+o r(\gamma)$.

Now let $g(\eta)=(\ulcorner\theta\urcorner \cdot 4+2)+o 1$.

Theorem 17. There is a function $h \in \mathcal{F}_{0}$ such that for $\eta \in \mathcal{I}_{1 p}$, if $W_{\eta}(\alpha, \beta)$ is well-founded then $h(\eta) \in O$, and $\Omega\left(W_{\eta}\right) \leq \Omega(h(\eta))$.

Proof. In the integer case, this is lemma I.4.3 of [15]. For $\eta \in \mathcal{I}_{1 p}$ and $\gamma<\kappa$ let $\tau(\eta, \gamma)=\left\ulcorner\phi_{\eta}\left(\xi_{1}, \xi_{2}\right) \wedge \xi_{1}<\dot{\gamma} \wedge \xi_{2}<\dot{\gamma}\right\urcorner$. Note that if $W_{\eta}$ is 
a nonempty well-founded relation then $\Omega\left(W_{\tau(\eta, \gamma)}\right)<\Omega\left(W_{\eta}\right)$ for all $\gamma$ and $\Omega\left(W_{\eta}\right)=\sup _{\gamma<\kappa} \Omega\left(W_{\tau(\eta, \gamma)}\right)+1$.

For $e \in \mathcal{I}_{1}$ and $\eta \in \mathcal{I}_{1 p}$ let $t(e, \eta)$ be the code for the formula in the free variable $\beta, \exists \gamma \exists \delta \phi_{\grave{\eta}}(\gamma, \delta) \wedge \exists \gamma \phi_{e}(\tau(\stackrel{\circ}{\eta}, \gamma), \beta)$.

Let $f \in \mathcal{F}_{1}$ be such that $W_{f(e)}=\{\langle\eta, g(t(e, \eta))\rangle\}$. Let $e_{0}$ be such that $W_{f\left(e_{0}\right)}=W_{e_{0}}$. Let $h$ be the function where $h(\eta)=g\left(t\left(e_{0}, \eta\right)\right)$. Then $W_{e_{0}}$ is the graph of $h$.

If $W_{e}$ is empty the theorem is readily seen. Otherwise $W_{t\left(e_{0}, \eta\right)}=\{h(\tau(\eta, \gamma))$ : $\gamma<\kappa\}$, and so by theorem 16 ,

$$
h(\tau(\eta, \gamma))<_{O} g\left(t\left(e_{0}, \eta\right)\right)=h(\eta)
$$

By induction on $\Omega\left(W_{\eta}\right), \Omega\left(W_{\tau(\eta, \gamma)}\right) \leq \Omega(h(\tau(\eta, \gamma)))<\Omega(h(\eta))$. Thus, $\Omega\left(W_{\eta}\right)=$ $\sup _{\gamma} \Omega\left(W_{\tau(\eta, \gamma)}\right)+1 \leq \Omega(h(\eta))$.

For $\beth=\nvdash, \nVdash, \nvdash$ let $O$ ב be the version of $O$ defined in Sections 7,6,5 respectively. For a class $C$ of relations let $C$-WF be the WF's of $C$, and similarly for $C$-WPS, $C$-WOS, and $C$-WO (by an earlier convention $\Upsilon(C$-WF $)$ is denoted $\Upsilon(C))$.

Theorem 18. $\quad$ a. $\Omega\left(O_{\nvdash}\right) \leq \Omega\left(O_{\nVdash}\right) \leq \Omega\left(O_{\nvdash}\right)$.

b. For $\beth=\nvdash, \nVdash, \nvdash \neq, \Omega\left(O_{\beth}\right), \Omega\left(C_{\beth-W O) \leq}\right.$

$\Omega\left(C_{\beth-} W O S\right) \leq \Omega\left(C_{\beth-W P S}\right) \leq \Upsilon\left(C_{\beth}\right)$.

c. $\Upsilon\left(C_{\nvdash}\right) \leq \Omega\left(O_{\nvdash}\right)$ and $\Omega\left(C_{\nvdash}-W O S\right) \leq \Omega\left(C_{\nvdash}-W O\right)$.

Proof. For part a, a $O_{\nvdash}$ code can be transformed to a $O_{\nVdash}$ code, and a $O_{\nVdash}$ code can be transformed to a $O_{\not \models}$ code.

For part b, $\Omega\left(O_{\beth}\right) \leq \Omega\left(C_{\beth}\right.$-WOS $)$ follows by theorems 14 and 12 . The other inequalities are immediate.

For part c, $\Upsilon\left(C_{\nvdash}\right) \leq \Omega\left(O_{\nvdash}\right)$ follows by theorem 17 .

Suppose $R(\alpha, \beta) \in C_{\nvdash}$-WOS. If $\Omega(R)<\kappa$ then clearly $\Omega(R)<\Omega\left(C_{\nvdash-W O}\right)$, so suppose $\Omega(R) \geq \kappa$. $\operatorname{Fld}(R)$ is defined by a formula $\exists \gamma \psi(\gamma, \beta, \vec{\pi})$ where $\psi$ is $\Delta_{0}$. Let $g: \kappa \mapsto \kappa$ be the function where $g(\alpha)=\beta$ iff there is an $s$ such that $\operatorname{Dom}(s)=\alpha$ and $s(\alpha)=\beta$ and $\forall \gamma \leq \alpha \psi\left(P_{1}(s(\gamma)), P_{2}(s(\gamma)), \vec{\pi}\right)$ and $\forall \gamma<\delta \leq \alpha\left(s\left(\gamma<\delta \wedge P_{2}(s(\gamma)) \neq s\left(P_{2}(\delta)\right)\right)\right)$. The relation $R(g(\alpha), g(\beta))$ is a WO of the same rank as $R$. Thus, $\Omega\left(C_{\nvdash}\right.$-WOS $) \leq \Omega\left(C_{\nvdash}\right.$-WO $)$.

As noted in Section 5, for suitable $\kappa, \Omega\left(O_{\not \models}\right)<\Omega\left(C_{\not \models-W O}\right)$ is consistent. Whether $\Omega\left(O_{\nVdash}\right)<\Omega\left(C_{\nVdash}\right.$-WO $)$ can be consistent is a question of interest. 


\section{Classes over $\kappa^{+}$}

For $\kappa \in$ Card, a class $C$ of Section 4 over $\kappa^{+}$will be denoted $\hat{C}$. Further classes of interest may be defined using second order methods over $\kappa$.

As in [8], let $L_{\in}^{s}$ denote $L_{\in}$ with set variables added, and let $L_{\in}^{f}$ denote $L_{\in}$ with function variables added. Recall $L_{\mathrm{OS}}^{f}$ from Section 3. Let $I_{\mathrm{OS}}$ denote the interpretation of $L_{\mathrm{OS}}$ in $L_{\in}^{s}$ given in [7]. Say that a formula is $\Sigma_{1}^{I}$ if it is the translation under $I_{\mathrm{OS}}$ of a $\Sigma_{1}$ formula of $L_{\mathrm{OS}}$. As in [9], let $\mathcal{N}_{g}$ denote $\left(V_{\kappa}\right)^{V_{\kappa}}$, let $\mathcal{N}$ denote $\kappa^{\kappa}$, and let $\Sigma_{1}^{1 L}$ denote the Lusin class in either $\mathcal{N}_{g}^{k}$ or $\mathcal{N}^{k}$ for an integer $k$.

The following class is defined.

$C_{\nsucceq}: D=\mathcal{N}, \Sigma_{1}^{1 P}$ in $L_{\mathrm{OS}}^{f}$ over $\mathrm{OS}_{\kappa}$.

Theorem 19. Suppose $\kappa \in$ Card. Then $\hat{C}_{\not \nVdash H} \rightsquigarrow C_{\not} \rightsquigarrow \hat{C}_{\not \neq}$. Thus, $\Upsilon\left(\hat{C}_{\not \models}\right)=\Upsilon\left(\hat{C}_{\not \neq}\right)=\Upsilon\left(\hat{C}_{\not \mathbb{H}}\right)=\Upsilon\left(C_{\not}\right)$.

Proof. Suppose $R \subseteq \mathcal{N}^{k}$ is defined in $H_{\kappa^{+}}$by a $\Sigma_{1}$ formula with parameters.

An element $F \in \mathcal{N}$ may be considered a binary relation $R$ on $\kappa$, where $R(\alpha, \beta)$ iff $F\left(J_{0}(\alpha, \beta)\right) \neq 0$. Recall from [7] that the Godel pairing function $J_{0}$ is $\Delta_{1}^{0}$. Given such a relation $F_{\in}$, the notation $\alpha \tilde{\epsilon} \beta$ will be used for $F_{\in}\left(J_{0}(\alpha, \beta)\right) \neq$ 0 .

Let $P_{1}\left(F_{\in}\right)$ hold iff as a binary relation $F_{\in}$ is well-founded and extensional. $F_{\in}$ is well-founded iff $\forall s(\operatorname{Dom}(s)=\omega \Rightarrow \exists n<\operatorname{Dom}(s)(s(n+1) \tilde{\notin} s(n))) . F_{\in}$ is extensional iff $\exists G \forall \alpha, \beta\left(\alpha \neq \beta \Rightarrow\left(G\left(J_{0}(\alpha, \beta)\right) \tilde{\notin} \alpha \Leftrightarrow G\left(J_{0}(\alpha, \beta)\right) \tilde{\epsilon} \beta\right)\right)$. Thus, $P_{1}$ is $\Sigma_{1}^{1 P}$.

A formula $\phi$ of the language of set theory, with value $\vec{\alpha}$ where $\alpha_{i}<\kappa$ for the free variables, can be coded as a value $\ulcorner\phi(\vec{\alpha})\urcorner$ which is less than $\kappa$. This can be done so that formulas defining predicates of interest are $\Delta_{1}^{0}$.

Let $P_{2}\left(F_{\in}, \alpha\right)$ hold iff $\phi$ is true in $F_{\in}$ where $\alpha=\ulcorner\phi\urcorner . P_{2}$ may be written as $\exists G_{1}, G_{2}\left(\forall \beta P_{2}^{\prime}\left(G_{1}, G_{2}, F_{\in}, \beta\right) \wedge G_{1}(\alpha) \neq 0\right)$ where $P_{2}^{\prime}$ is a $\Delta_{1}^{0}$ formula stating that $G_{1}(\beta)$ satisfies the recursion for a truth value assignment to the sentence with Godel number $\beta$. This may be broken into cases. Most of these are given in example 1.20 of [14]. The case $\beta=\ulcorner\exists x \psi\urcorner$ may be written as $G_{1}(\beta) \neq 0 \Leftrightarrow$ $G_{1}\left(\left\ulcorner\psi_{G_{2}(\beta) / x}\right\urcorner\right) \neq 0$. Thus, $P_{2}$ is $\Sigma_{1}^{1 P}$.

The notation $\llbracket \phi \rrbracket$ will be used for $P_{2}\left(F_{\in},\ulcorner\phi\urcorner\right)$.

For $X \in \mathcal{N}$ let $P_{3}\left(F_{\in}, X, \alpha\right)$ hold iff $\alpha$ represents $X$ in $F_{\in}$. Following lemma 1.25 of [14], let $P_{3}^{1}\left(F_{\epsilon}, F_{\kappa}, \alpha_{\kappa}\right)$ be the $\Sigma_{1}^{1 P}$ formula $\exists F_{\kappa}^{r}(\forall \alpha, \beta(\alpha<\beta \Rightarrow$ $\left.\left.F_{\kappa}(\alpha) \tilde{\epsilon} F_{\kappa}(\beta)\right) \wedge \forall \alpha\left(F_{\kappa}(\alpha) \tilde{\epsilon} \alpha_{\kappa}\right) \wedge \forall \alpha\left(\alpha \tilde{\epsilon} \alpha_{\kappa} \Rightarrow F_{\kappa}\left(F_{\kappa}^{r}(\alpha)\right)=\alpha\right)\right)$. Then $P_{3}$ may be written as $\exists F_{\kappa}, \alpha_{\kappa}\left(P_{3}^{1}\left(F_{\in}, F_{\kappa}, \alpha_{\kappa}\right) \wedge \llbracket \stackrel{\alpha}{\alpha}: \stackrel{\circ}{\alpha}_{\kappa} \mapsto \stackrel{\circ}{\alpha}_{\kappa} \rrbracket \wedge \forall \beta, \gamma(X(\beta)=\gamma \Rightarrow\right.$ 


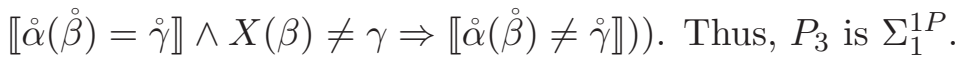

An element $p \in H_{\kappa^{+}}$can be coded as an element $P \in N$ by enumerating the transitive closure of $\{p\}$ as $x_{\alpha}$ and letting $P\left(J_{0}(\alpha, \beta)\right) \neq 0$ iff $x_{\alpha} \in$ $x_{\beta}$. Let $P_{4}\left(F_{\in}, P, \alpha\right)$ hold iff $\alpha$ represents $P$ in $F_{\in}$. $P_{4}$ may be written as $\exists G \exists \beta\left(\forall \gamma, \delta\left(P_{\tilde{\epsilon}}\left(J_{0}(\gamma, \delta)\right) \neq 0 \Leftrightarrow(G(\gamma) \tilde{\epsilon} \beta \wedge G(\delta) \tilde{\epsilon} \beta \wedge G(\gamma) \tilde{\in} G(\delta))\right) \wedge \alpha \tilde{\epsilon} \beta \wedge\right.$ $\forall \gamma(\gamma \tilde{\in} \beta \Rightarrow \alpha \tilde{\notin} \gamma))$. Thus, $P_{4}$ is $\Sigma_{1}^{1 P}$.

Suppose $\phi(\vec{X}, \vec{p})$ is a $\Sigma_{1}$ formula, the $X_{i}$ are restricted to range over $\mathcal{N}$, and the $p_{i}$ are elements of $H_{\kappa^{+}}$. Using the Downward Lowenheim-Skolem theorem, $\phi$ holds in $H_{\kappa^{+}}$iff (using obvious notation) $\exists F_{\in}, \vec{\alpha}_{X}, \vec{\alpha}_{p}\left(P_{1}\left(F_{\epsilon}\right) \wedge P_{3}\left(F_{\epsilon}, \vec{X}, \vec{\alpha}_{X}\right) \wedge\right.$ $\left.P_{4}\left(F_{\in}, \vec{P}, \vec{\alpha}_{p}\right) \wedge P_{2}\left(F_{\epsilon},\left\ulcorner\phi\left(\vec{\alpha}_{X}, \vec{\alpha}_{p}\right)\right\urcorner\right)\right)$ holds in $\operatorname{OS}_{\kappa}^{f}$.

Thus, $R$ is defined in $\operatorname{OS}_{\kappa}$ by a $\Sigma_{1}^{1 P}$ formula with second order parameters. and so $\hat{C}_{\not \neq \mathbb{H}} \rightsquigarrow C_{\not}$.

$C_{\not} \rightsquigarrow \hat{C}_{\not}$ follows because there is an interpretation of $L_{\mathrm{OS}}^{f}$ in $L_{\mathrm{OS}}$ using the parameter $\kappa$ which induces such a transformation. Ord is interpreted as $\kappa$, Seq is interpreted as $\{s \in \operatorname{Seq}: \operatorname{Dom}(s)<\kappa \wedge \operatorname{Ran}(s) \subseteq \kappa\} . \mathcal{N}$ is interpreted as $\{s \in \operatorname{Seq}: \operatorname{Dom}(s)=\kappa \wedge \operatorname{Ran}(s) \subseteq \kappa\}$.

The second claim follows by the first claim and theorem 7 .

For a $\Sigma_{1}^{1 L}$ version of $\hat{C}_{\nvdash \mathbb{H}} \rightsquigarrow C_{\not}$ see proposition 2.4 of [13].

Theorem 20. Suppose $\kappa \in$ Card. Then $\hat{C}_{\nVdash \mathbb{H}} \rightsquigarrow \hat{C}_{\nVdash}$, and so $\Upsilon\left(\hat{C}_{\nVdash \mathbb{H}}\right)$ $=\Upsilon\left(\hat{C}_{\nVdash}\right)$,

Proof. The theorem is proved by modifying the composite

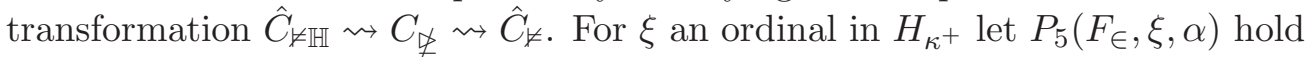
iff $\alpha$ represents $\xi$ in $F_{\in}$. This may be written as $\exists s_{o}, s_{o}^{r}(\operatorname{Dom}(s)=\xi \wedge \forall \beta<$ $\operatorname{Dom}(s)\left(s_{o}(\beta) \tilde{\epsilon} \alpha\right) \wedge \forall \beta, \gamma<\xi\left(\beta<\gamma \Rightarrow s_{o}(\beta) \tilde{\epsilon} s_{o}(\gamma)\right) \wedge \forall \beta\left(\beta \tilde{\epsilon} \alpha \Rightarrow s_{o}\left(s_{o}^{r}(\beta)\right)=\right.$ $\beta))$. Thus, $P_{5}$ is $\Sigma_{1}$ over $\mathrm{OS}_{\kappa^{+}}$.

Suppose $\phi(\vec{\xi}, \vec{p})$ is a $\Sigma_{1}$ formula over $H_{\kappa^{+}}$, the $\xi_{i}$ are restricted to range over ordinals, and the $p_{i}$ are ordinals. Then $\phi$ holds in $H_{\kappa^{+}}$iff $\exists F_{\in}, \vec{\alpha}_{\xi}, \vec{\alpha}_{p}\left(P_{1}\left(F_{\in}\right) \wedge\right.$ $\left.P_{5}\left(F_{\epsilon}, \vec{\xi}, \vec{\alpha}_{\xi}\right) \wedge P_{5}\left(F_{\epsilon}, \vec{p}, \vec{\alpha}_{p}\right) \wedge P_{2}\left(F_{\epsilon},\left\ulcorner\phi\left(\vec{\alpha}_{\xi}, \vec{\alpha}_{p}\right)\right\urcorner\right)\right)$ holds in $\mathrm{OS}_{\kappa^{+}}$. This shows $\hat{C}_{\nVdash \bar{\sim}} \rightsquigarrow \hat{C}_{\nVdash}$.

The second claim follows from the first, and theorem 7 .

As noted in [9] it is consistent that $\Upsilon\left(\hat{C}_{\not z}\right)>\Upsilon\left(\hat{C}_{\nVdash}\right)$. By theorem 1.1 of [12], if $\kappa$ is a regular uncountable cardinal with $\kappa^{<\kappa}=\kappa$ then it is consistent that there is a $\Sigma_{1}$ well-order of $H_{\kappa^{+}}$of order type $\geq \kappa^{++}$, whence that there is a $\Sigma_{1}$ WOS with field $\kappa^{\kappa}$ of rank $\geq \kappa^{++}$.

For $\kappa \in$ Card, the following classes of relations are defined.

$C_{\not}: D=\left(V_{\kappa}\right)^{V_{\kappa}}, \Sigma_{1}^{1}$ in $L_{\in}^{f}$ over $V_{\kappa}$. 
$C_{\not}: D=\mathcal{N}_{g}, \Sigma_{1}^{1 L}$.

$C_{\not}: D=\mathcal{N}, \Sigma_{1}^{1 L}$.

$C_{\nleftarrow}: D=I_{\text {Seq }}, \Sigma_{1}^{I}$ over $V_{\kappa}$.

$C_{\nrightarrow}: D=2^{V_{\kappa}}, \Sigma_{1}^{1}$ in $L_{\in}^{s}$ over $V_{\kappa}$.

Say that $C \stackrel{I}{\rightsquigarrow} C^{\prime}$ provided $\kappa$ is inaccessible, and $C \stackrel{B}{\rightsquigarrow} C^{\prime}$ provided $\kappa$ is regular uncountable and $\kappa^{<\kappa}=\kappa$.

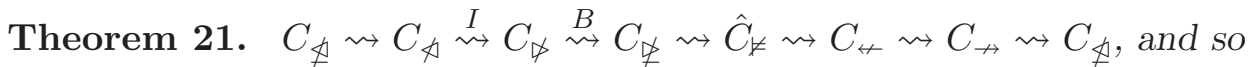
for $\kappa \in \operatorname{Inac}, \Upsilon\left(C_{\nexists}\right)=\Upsilon\left(C_{\nexists}\right)=\Upsilon\left(C_{\not}\right)=\Upsilon\left(C_{\not}\right)=\Upsilon\left(\hat{C}_{\not z}\right)=\Upsilon\left(C_{\sharp}\right)=\Upsilon\left(C_{\nrightarrow}\right)$.

Proof. Suppose $R(\vec{H})$ is a relation in $C_{\Varangle}$; by corollary 5 of [8] $R$ is defined by a formula $\exists \vec{F} \forall \vec{x} \psi(\vec{x}, \vec{F}, \vec{G}, \vec{H})$ where $\psi$ is a $\Delta_{0}^{0}$ formula of $L_{\in}^{f}$ and $\vec{G}$ are second order parameters. By theorem 9 of [9] and remarks following $R(\vec{H})$ is $C_{\text {丸 }}$. Hence $C_{\text {丸力 }} \rightsquigarrow C_{\Varangle}$.

Recall from [9] the homeomorphism $\hat{E}: \mathcal{N} \mapsto \mathcal{N}_{g}$ derived from a bijection $E: \kappa \mapsto V_{\kappa}$ for $\kappa \in$ Inac. Using this, a $C_{丸}$ relation $R(\vec{H})$ may be transformed to a $C_{\not}$ relation $R\left(\vec{H}^{\hat{E}}\right)$. Hence $C_{\not} \stackrel{I}{\rightsquigarrow} C_{\not}$.

Suppose $R(\vec{H})$ is a relation in $C_{\not}$, being the projection along $\vec{F}$ of the closed subset $K(\vec{F}, \vec{H})$. As in the proof of theorem 5.b, $K^{c}$ can be specified by a subset $D_{K^{c}}$ of $\mathrm{Seq}_{\kappa}^{k+l}$. Since $\kappa^{<\kappa}=\kappa D_{K^{c}}$ can be coded as an element $G$ of $\mathcal{N}$ using a "separator" value. $R$ may be defined in $\mathrm{OS}_{\kappa}$ by the $\Sigma_{1}^{1 P}$ formula with the parameter $G$, "there is a $\vec{F}$ such that no element of $G$ which is a prefix of $\langle\vec{F}, \vec{H}\rangle$ ". Hence $C_{\not} \stackrel{B}{\rightsquigarrow} \hat{C}_{\not \neq}$.

$C_{\nsucceq} \rightsquigarrow \hat{C}_{\not}$ is proved in theorem 19.

Suppose $R(\vec{\alpha}, \vec{s})$ is a relation in $\hat{C}_{\not}$, defined by $\Sigma_{1}$ formula $\phi(\vec{\beta}, \vec{t}, \vec{\alpha}, \vec{s})$ with parameters $\vec{\beta}, \vec{t}$. Let $\phi_{I}$ be the interpretation under $I_{\mathrm{OS}}$; this defines a relation on $I_{\text {Ord }}^{k} \times I_{\text {Seq }}^{l}$ for appropriate $k, l$. In particular $\hat{C}_{\not \models} \rightsquigarrow C_{\nleftarrow}$. (This stretches the definition of $\rightsquigarrow$, but WF's transform to WF's and WPS's to WPS's).

It follows using results of [7] that $C_{\nleftarrow} \rightsquigarrow C_{\nrightarrow}$.

It follows using lemma 3 of [8] that $C_{\nrightarrow} \rightsquigarrow C_{\text {丸 }}$.

That $\Upsilon\left(C_{\ngtr}\right)=\Upsilon\left(C_{\not}\right)$ for $\kappa \in$ Inac can be improved.

Theorem 22. $C_{\ngtr} \rightsquigarrow C_{\psi} \stackrel{B}{\rightsquigarrow} C_{\ngtr}$, and so for a regular uncountable cardinal $\kappa$ such that $\kappa^{<\kappa}=\kappa, \Upsilon\left(C_{\notin}\right)=\Upsilon\left(C_{\not}\right)$.

Proof. This follows by lemma 5 . 
As seen in [7], the class $C_{\nrightarrow}$ is of interest in connection with function and set chains. The class $\hat{C}_{\not \models}$ provides a first-order characterization. The class $\hat{C}_{\not \models \mathbb{H}}$ has already been considered (in [13] for example). $\hat{C}_{\not \models}$ has an advantage over $\hat{C}_{\not \models \mathbb{H}}$, in that the transformation $\hat{C}_{\not \models} \rightsquigarrow \hat{C}_{\nleftarrow}$ provides a structured interpretation of the first-order formulas in the second-order ones.

There is an interpretation of $H_{\kappa^{+}}$in $L_{\mathrm{OS}}^{f}$. The domain is the set of $F \in \mathcal{N}$, which as binary relations are well-founded, extensional, transitive, and have a maximal element. The interpretations of $\in$ and $=$ are $\Sigma_{1}^{1 P}$.

\section{0. $\Delta_{1}$ Classes}

For any of the classes of relations $C$ of Sections 4 and 9 , say that $R \in C$ is in class $C^{\Delta}$ if $R$ has a $\Pi_{1}$ definition also, where $\Pi_{1}$ is defined appropriately.

Theorem 23. a. The transformations of theorems 7, 19, 20, and 21 map $\Delta$ relations to $\Delta$ relations.

b. For classes $C_{\beth}$ for $\beth=\nvdash, \nVdash, \nvdash, \nVdash$, if $\preceq \in C_{\mathbb{I}}$ is a total order then $\preceq \in C_{\beth}$.

c. $\Upsilon\left(C_{\nvdash}\right)=\Upsilon\left(C_{\nvdash}^{\Delta}\right)$.

Proof. Part a follows by additional observations in the proofs of the cited theorems. Part b follows by the usual proof. Part c follows by part b and theorem 18.

\section{Function Chains}

Suppose $\kappa$ is a regular uncountable cardinal. For $f, g: \kappa \mapsto \kappa$ say that $f \leq_{\mathrm{t}} g$ if $\{\alpha<\kappa: f(\alpha)<g(\alpha)\}$ is in the club filter, and similarly for $f<_{\mathrm{t}} g$ and $f \equiv_{\mathrm{t}} g$. As noted in [7], if $\kappa \in \operatorname{Inac} f, g$ need only be defined for $\alpha \in$ Card. A function chain is a chain in this order.

As also noted in [7], if $\kappa$ is Mahlo, $f, g$ defined only for $\alpha \in$ Inac may be considered. As far as the author knows, it is unknown whether the lengths of the function chains in the order when the domain is Inac are no greater than those when the domain is Card.

$C_{\nrightarrow}$ will also be denoted $\Sigma_{1}^{1}$. Let $\Sigma_{1 l^{1}}^{1}$ WPS denote the $\Sigma_{1}^{1}$ WPS's $\preceq$ such that $\prec$ is also $\Sigma_{1}^{1}$. For $\kappa \in$ Inac Let $\mathcal{U} \Sigma_{1 l}^{1}$-WPS denote the $\Sigma_{1}^{1}$ WPS's $\preceq$, such that the formulas define a WPS and its strict part, at any inaccessible cardinal below $\kappa$ as well (these are denoted $\mathcal{U}_{\Sigma_{1}^{1}}$ in [7]). As in [7], for such $\preceq$, for $\boldsymbol{\alpha} \in \operatorname{Fld}(\preceq)$, the function $f_{\boldsymbol{\alpha}}: \operatorname{Inac}_{\kappa} \mapsto \kappa$ is that where $f_{\boldsymbol{\alpha}}(\lambda)=\Omega\left(\preceq_{\lambda, \boldsymbol{\alpha} \cap V_{\lambda}}\right)$. Let $\mathcal{C}_{I}$ denote 
the filter $\{C \cap$ Inac : $C \subseteq \kappa$ is club $\}$. Theorem 13 of [7] states that for $\kappa$ a Mahlo cardinal, if $\boldsymbol{\alpha} \preceq \boldsymbol{\beta}$ then $f_{\boldsymbol{\alpha}} \leq_{\mathcal{C}_{I}} f_{\boldsymbol{\beta}}$, and if $\boldsymbol{\alpha} \prec \boldsymbol{\beta}$ then $f_{\boldsymbol{\alpha}}<_{\mathcal{C}_{I}} f_{\boldsymbol{\beta}}$.

Suppose $\kappa \in$ Inac. A $\mathcal{R} \Sigma_{1}^{1}$ specification of a WF $\prec$ is a pair $\langle\phi, C\rangle$ where $\phi(X, Y, \vec{P})$ is a $\Sigma_{1}^{1}$ formula with class parameters $P_{i} \subseteq V_{\kappa}$, and $C \subseteq \kappa$ is a club, such that $\phi$ defines a WF $\prec$ in $V_{\kappa}$ and a WF $\prec_{\lambda}$ in $V_{\lambda}$ for $\lambda \in C$. A $\mathcal{R} \Sigma_{1}^{1} \mathrm{WF}$ is one for which there is a $\mathcal{R} \Sigma_{1}^{1}$ specification.

If $\kappa \in$ Inac and $\preceq$ is a $\mathcal{R} \Sigma_{1}^{1}$ WF let $C$ be the club of the specification, and for $\boldsymbol{\alpha} \in \operatorname{Fld}(\preceq)$ let $f_{\boldsymbol{\alpha}}:$ Card $\cap \kappa \mapsto \kappa$ be the function where for $\lambda \in$ Card, if $\lambda \in C$ then $f_{\boldsymbol{\alpha}}(\lambda)=\Omega\left(\preceq_{\lambda, \boldsymbol{\alpha} \cap V_{\lambda}}\right)$, else $f_{\boldsymbol{\alpha}}(\lambda)=0$.

Theorem 24. Suppose $\kappa \in$ Inac and $\prec$ is a $\mathcal{R} \Sigma_{1}^{1} W F$. Let $C_{1}$ be the club in the specification of $\prec$. If $\boldsymbol{\alpha} \prec \boldsymbol{\beta}$ then there is a club $C_{2} \subseteq C_{1}$ such that for $\lambda \in C_{2}, \boldsymbol{\alpha} \cap V_{\lambda} \prec_{\lambda} \boldsymbol{\beta} \cap V_{\lambda}$; in particular $f_{\boldsymbol{\alpha}}<_{t} f_{\boldsymbol{\beta}}$.

Proof. Let $C$ be as in the proof of theorem 13.b of [7]. Let $C_{2}=C_{1} \cap C$.

It follows that if $\prec$ is a $\mathcal{R} \Sigma_{1}^{1} \mathrm{WF}$ then for any $\alpha<\Upsilon(\prec)$ there is a chain of length $\alpha$ in the order $<_{\mathrm{t}}$. If $\kappa \in$ Inac and $V=L$ then by theorems $21,17,14$ for $L_{\kappa^{+}}$, and the fact that the interpretation under $I_{\mathrm{OS}}$ of a WOS is a WPS, $\Upsilon\left(\Sigma_{1}^{1}\right)=\Upsilon\left(\Sigma_{1}^{1}\right.$-WPS $)$. It is a question of interest whether, under the same hypotheses, $\Upsilon\left(\mathcal{R} \Sigma_{1}^{1}\right)=\Upsilon\left(\mathcal{R} \Sigma_{1}^{1}\right.$-WPS $)$.

Recall from [7] the definition of $f<\mathcal{F} g$ on $\kappa^{D}$ for a domain $D$ and a filter $\mathcal{F}$ of subsets of $D . D=\operatorname{Card} \cap \kappa$ and $\mathcal{F}=\mathcal{C}$ where $\mathcal{C}$ is the club filter, and $D=$ Inac $\cap \kappa$ and $\mathcal{F}=\mathcal{C}_{I}$ where $\mathcal{C}_{I}=\{$ Inac $\cap C: C \in \mathcal{C}\}$, are examples of interest. A function defined on $E \in \mathcal{F}$ may be extended to $D$ by setting the value to 0 on $D-E$.

Suppose $\kappa$ is weakly compact. Let $\mathcal{E}$ denote the enforceable filter, and let $D=$ Card. Suppose $\prec$ is a $\Sigma_{1}^{1}$ WF. Since the statement that $\prec$ is well-founded is $\Pi_{1}^{1}$, there is an $E \in \mathcal{E}$ such that for $\lambda \in \mathcal{E}, \prec_{\lambda}$ is well-founded.

Theorem 25. Suppose $\kappa$ is weakly compact and $\prec$ is a $\Sigma_{1}^{1} W F$. Let $E_{1} \in \mathcal{E}$ be such that $\prec_{\lambda}$ is well-founded for $\lambda \in \mathcal{E}$. If $\boldsymbol{\alpha} \prec \boldsymbol{\beta}$ then there is an $E_{2} \in \mathcal{E}$ with $E_{2} \subseteq E_{1}$ such that for $\lambda \in E_{2}, \boldsymbol{\alpha} \cap V_{\lambda} \prec_{\lambda} \boldsymbol{\beta} \cap V_{\lambda}$; in particular $f_{\boldsymbol{\alpha}}<_{\mathcal{E}} f_{\boldsymbol{\beta}}$.

Proof. Let $C$ be as in the proof of theorem 13.b of [7]. Let $E_{2}=E_{1} \cap C$.

\section{Set Chains}

Recall from [7] that for $\kappa \in$ Inac and $X, Y \subseteq \kappa, X \subseteq_{\mathrm{t}} Y$ if $X-Y$ is thin. For $X \subseteq \operatorname{Inac}_{\kappa}$ let $\mathrm{H}(X)=\{\lambda \in X: X \cap \lambda$ is a stationary subset of $\lambda\}$. For $X, Y$ 
stationary subsets of $\operatorname{Inac}_{\kappa}$ say that $X<_{R} Y$ if $Y \subseteq_{\mathrm{t}} \mathrm{H}(X)$. This relation is transitive and well-founded; let $\rho_{R}$ denote the rank function. Note that $<_{R}$ is empty unless $\kappa$ is Mahlo. By a set chain is meant a chain in this order.

Set chains for $\mathcal{U} \Sigma_{1 l^{1}}^{1}$ WPS's were defined in [7]. Modifying the development as necessary, set chains for $\mathcal{R} \Sigma_{1}^{1}$ WF's may be defined.

Indeed, suppose $\prec$ is a $\mathcal{R} \Sigma_{1}^{1} \mathrm{WF}$ with $C$ the club of the specification. For $\boldsymbol{\alpha} \in \operatorname{Fld}(\prec)$ and $X \subseteq \operatorname{Inac}_{\kappa} \cap C$, say that $\lambda \in \mathrm{H}^{\alpha}(X)$ iff $\lambda \in X$ and $\mathrm{H}^{\gamma}(X \cap \lambda)$ is a stationary subset of $\lambda$ for all $\gamma \in \operatorname{Fld}\left(\prec_{\lambda}\right)$ where $\gamma<f_{\boldsymbol{\alpha}}(\lambda)$, or equivalently $\gamma \prec_{\lambda} \boldsymbol{\alpha} \cap V_{\lambda}$.

Theorem 26. Suppose $\kappa \in$ Inac and $\prec$ is a $\mathcal{R} \Sigma_{1}^{1} W F$ with $C$ the club of the specification.

a. If $\beta \leq \alpha$ then for any $X \subseteq \operatorname{Inac}_{\kappa} \cap C, H^{\beta}(X) \supseteq_{t} H^{\alpha}(X)$.

b. Suppose $\boldsymbol{\alpha} \in$ Fld $(\prec), X \subseteq \operatorname{Inac}_{\kappa} \cap C$, and $\lambda \in \operatorname{Inac}_{\kappa} \cap C$. Then $H^{\alpha \cap V_{\lambda}}(X \cap$ $\lambda)=H^{\alpha}(X) \cap \lambda$.

c. If $\beta<\alpha$ then for any $X \subseteq \operatorname{Inac}_{\kappa} \cap C, H\left(H^{\beta}(X)\right) \supseteq_{t} H^{\alpha}(X)$.

Proof. Part a is like theorem 14 of [7], part b is like lemma 15, and part c is like theorem 16 . The proofs are almost unchanged.

Theorem 27. Suppose $\kappa \in$ Inac and $\prec$ is a $\mathcal{R} \Sigma_{1}^{1} W F$ with $C$ the club of the specification.

a. There is a $\Pi_{1}^{1}$ formula $\Phi_{\prec}(A)$ which holds in $V_{\kappa}$ iff $H^{A}($ Inac) is stationary.

b. If $\kappa$ is weakly compact then $\models_{V_{\kappa}} \Phi_{\prec}(A)$ for any $A \in \operatorname{Fld}(\prec)$.

Proof. Lemma 18, theorem 19, and theorem 20 of [7] hold, with suitable modifications. $\preceq$ is replaced by $\prec$. $\phi$ is not used, and $\psi$ is replaced by $\phi$. Inac is replaced by $\operatorname{Inac} \cap C$. In the proof of theorem 20, $\operatorname{Fld}(\prec)$ is $\Sigma_{1}^{1}$, being defined by $\exists Y(X \prec Y \vee Y \prec X)$; a similar modification is made to the proof of lemma 18.

Adapting the discussion of axiom $\mathcal{U}_{\Delta_{\infty}^{1}}$ WPS's in [7], suppose $\phi(X, Y, \vec{P}, C)$ is a formula of $L_{\epsilon}^{s}$. The statement that $\phi$ defines a WF, and does so in $V_{\kappa}$ for any $\kappa \in$ Card $\cap C$, may be expressed by a formula $\Psi_{\phi}$ of $L_{\in}^{s}$ (the parameters being free variables), which will also be denoted $\Psi_{\prec}$. The formula $\Phi_{\prec}(A)$ of theorem 19 of [7], adapted to $\mathcal{R} \Sigma_{1}^{1}$ WF's, may be given for any WF and not just $\Sigma_{1}^{1}$ WF's, although $\Phi_{\prec}$ is no longer $\Pi_{1}^{1}$ (this observation was omitted in [7]). The axiom $\mathrm{Ax}_{\prec}$ is then $\Psi_{\prec} \Rightarrow \forall A \Phi_{\prec}(A)$. This axiom may be justified as the axiom $A_{\preceq}$ of [7].

Say that a cardinal is $\mathcal{R} \Delta_{\infty}^{1}$-Mahlo if Ax $\prec$ holds in $V_{\kappa}$ for all $\Delta_{\infty}^{1}$ WF's $\prec$ in $V_{\kappa}$. The axiom stating that these cardinals exist is justified by collecting the 
universe; by fairly strict standards, these cardinals are "built up". Say that a cardinal $\kappa$ is $\mathcal{R} \Sigma_{1}^{1}$-Mahlo if $\mathrm{Ax}_{\prec}$ holds in $V_{\kappa}$ for all $\mathcal{R} \Sigma_{1}^{1} \mathrm{WF}$ 's $\prec$ in $V_{\kappa}$. By theorem 27 a weakly compact cardinal is $\mathcal{R} \Sigma_{1}^{1}$-Mahlo.

There is a "gap" between $\mathcal{R} \Sigma_{1}^{1}$-Mahlo cardinals and weakly compact cardinals. Closing this gap provides a specific method for attempting to build up a weakly compact cardinal.

\section{References}

[1] J. Barwise, Admissible Sets and Structures, Springer-Verlag, Berlin, 1971.

[2] M. Dowd, Iterating Mahlo's operation, Int. J. Pure Appl. Math. bf 9, no. 4 (2003), 469-512. http://www.hyperonsoft.com/imol.pdf

[3] M. Dowd, A Lower Bound on the Mahlo Rank of a Weakly Compact Cardinal, Int. J. Pure Appl. Math. 68, no. 4 (2011), 415-438. http://www.ijpam.eu/contents/2011-684/4/4.pdf

[4] M. Dowd, Improved Results in Scheme Theory, Int. J. Pure Appl. Math. 76, No. 2 (2012), 173-190. http://ijpam.eu/contents/2012-76-2/3/3.pdf

[5] M. Dowd, Scheme Terms, Int. J. Pure Appl. Math. 81, no. 1 (2012), 111-128. http://www.ijpam.eu/contents/2012-81-1/11/11.pdf

[6] M. Dowd, New Constructions of Function and Set Chains, Int. J. Pure Appl. Math. 89, no. 3 (2013), 377-392. http://dx.doi.org/10.12732/ijpam.v89i3.9

[7] M. Dowd, Function Chains from Uniform Sigma-1-1 Well Orders, Int. J. Pure Appl. Math. 93 no. 5 (2014), 661-684.

http://dx.doi.org/10.12732/ijpam.v93i5.7

[8] M. Dowd, Pi-1-1 Normal Form in a Regular Cardinal, Int. J. Pure Appl. Math. 96, no. 1 (2014), 135-142. http://dx.doi.org/10.12732/ijpam.v96i1.10

[9] M. Dowd, Downsets and Generalized Descriptive Set Theory, Int. J. Pure Appl. Math. 101, no. 2 (2015), 305-324. http://dx.doi.org/10.12732/ijpam.v101i2.15

[10] F. Drake, Set Theory, An Introduction to Large Cardinals, North Holland (1974).

[11] P. Hajek and P. Pudlak, Metamathematics of first-order arithmetic, Springer-Verlag, Berlin, 1993.

[12] P. Holy and P. Lucke, Locally $\Sigma_{1}$-Definable Well-Orders of $\mathrm{H}\left(\mathrm{K}^{+}\right)$, Fund. Math. 226 (2014), 221-236. doi:10.4064/fm226-3-2

[13] P. Lucke, $\Sigma_{1}^{1}$-definability at uncountable regular cardinals, J. Symbolic Logic $\mathbf{7 7}$ no. 3 (2012), 1011-1046. http://dx.doi.org/10.2178/jsl/1344862172 
[14] R. Mansfield and G. Weitkamp, Recursive Aspects of Descriptive Set Theory, Oxford Logic Guides 11, 1985.

[15] G. Sacks, Higher Recursion Theory, Springer-Verlag, Berlin, 1990.

[16] D. Schmidt, The Relation between the Height of a Well-Founded Partial Ordering and the Order Types of Its Chains and Antichains, Journal of Combinatorial Theory, Series B 31 (1981), 183-189. 
